\title{
Estudio Fitoquímico y Evaluación de la Actividad Antioxidante y Leishmanicida de la Especie Pilocarpus alvaradoi (Rutaceae)
}

\author{
Fernis J. Marin ${ }^{(1,2)}$, Omar L. Torres ${ }^{(1,2) \star}$, Sara M. Robledo ${ }^{(3)}$ y Maria E. Doria ${ }^{(1,2)}$ \\ (1) Facultad de Ciencias de la Salud, Dpto. de Regencia y Farmacia, Univ. de Córdoba, Montería- Colombia \\ (e-mail: fernismarin@hotmail.com; omart2365@gmail.com; mayyieugen1212@hotmail.com) \\ (2) Facultad de Ciencias, Dpto. de Química, Univ. de Córdoba, Carrera 6 No 76-103, Montería- Colombia \\ (3) Instituto de Investigaciones Médicas. Fac. de Medicina, Universidad de Antioquia, Calle 62 No. 52-59 \\ SIU Laboratorio 632, Medellín-Colombia (e-mail: sara.robledo@udea.edu.co)
}

* Autor a quien debe ser dirigida la correspondencia

Recibido Ago. 10, 2017; Aceptado Nov. 3, 2017; Versión final Ene. 27, 2018, Publicado Abr. 2018

\begin{abstract}
Resumen
Se realizó el estudio fitoquímico y se evaluó la actividad antioxidante y leishmanicida de la especie Pilocarpus alvaradoi. La identificación de los compuestos se realizó mediante el análisis de datos de $\mathrm{RMN}-{ }^{1} \mathrm{H},{ }^{13} \mathrm{C}$ y EM. La actividad antioxidante se evaluó usando los métodos DPPH y ABTS ${ }^{++}$; el potencial leishmanicida in vitro fue medido frente a cepas de Leishmania $(V)$ panamensis mediante citometría de flujo y la citotoxicidad por el método MTT. De esta especie se aislaron e identificaron seis compuestos: una cumarina, un alcaloide, tres esteroles y un ácido graso. El extracto etanólico de corteza mostró baja citotoxicidad con una $\mathrm{CL}_{50}$ de 70.5 $\mu \mathrm{g} / \mathrm{ml}$ y un buen potencial leishmanicida con una $\mathrm{CE}_{50}$ de $30.7 \mu \mathrm{g} / \mathrm{ml}$ comparado con la Anfotericina B. Los extractos de Pilocarpus alvaradoi constituyen una importante fuente de metabolitos con actividad biológica; y las sustancias obtenidas son reportadas por primera vez para esta especie.
\end{abstract}

Palabras clave: Pilocarpus; actividad leishmanicida; antioxidante; leishmania; Rutaceae

\section{Phytochemical Study and Evaluation of Antioxidant and Leishmanicidal Activity of the Species Pilocarpus alvaradoi (Rutaceae)}

\begin{abstract}
The phytochemical study was carried out and the antioxidant and leishmanicidal activity of the Pilocarpus alvaradoi species was evaluated. Identification of the compounds was performed by the analysis of NMR- ${ }^{1} \mathrm{H}$, ${ }^{13} \mathrm{C}$ and MS data. Antioxidant activity was assessed using the $\mathrm{DPPH}^{\cdot}$ and $\mathrm{ABTS}{ }^{\cdot+}$ methods; the leishmanicidal potential in vitro was measured against strains of Leishmania (V) panamensis by flow cytometry and cytotoxicity by the MTT method. Of this species, six compounds were isolated and identified: a coumarin, an alkaloid, three sterols and a fatty acid. The ethanolic bark extract showed low cytotoxicity with a $\mathrm{CL}_{50}$ of 70.5 $\mu \mathrm{g} / \mathrm{ml}$ and a good leishmanicidal potential with an $\mathrm{EC}_{50}$ of $30.7 \mu \mathrm{g} / \mathrm{ml}$ compared to amphotericin $\mathrm{B}$. The extracts of Pilocarpus alvaradoi constitute an important source of metabolites with biological activity; and the substances obtained are reported for the first time for this specie.
\end{abstract}

Keywords: Pilocarpus; leishmanicidal activity; antioxidant; leishmanial; Rutaceae 


\section{INTRODUCCIÓN}

La familia Rutáceae está constituida por un amplio número de plantas, y una variada aplicabilidad en el campo de la medicina tradicional, usados como febrífugo, diurético, analgésico, para aliviar afecciones gastrointestinales, ulceraciones y para el tratamiento contra mordeduras de serpientes (Coy et al., 2013). En Colombia se han identificado 20 géneros de esta familia; algunas especies pertenecientes a estos géneros han mostrado una amplia variedad de actividades biológicas como: antiinflamatoria (Shen et al., 2014), anticancerígena, antimalárica, antioxidante, anti-VIH y antimicrobiana (Uvarani et al., 2013). Este amplio rango de actividades biológicas se atribuye a la presencia de metabolitos secundarios con diversidad estructural contenidas en las diferentes especies de esta familia como son los alcaloides (Uvarani et al., 2013; Beniddir et al., 2014), limonoides (Xia et al., 2014), dihidrochalconas (De Melo et al., 2014), cumarinas (Lin et al., 2014) y flavonoides (Peng et al., 2012). Investigaciones realizadas sobre especies pertenecientes al género Pilocarpus (Rutaceae) muestran la identificación de metabolitos secundarios activos como: limoneno, undecanona y sabineno, obtenidos como aceite esencial de las partes aéreas de Pilocarpus spicatus SaintHilaire, el cual presentó actividad anticolinesterasa, efecto inhibitorio sobre el crecimiento bacteriano ( $E$. coli y S. aureus), pero resultó ser tóxico para las larvas de Artemia salina (Oliveira et al., 2010); de las hojas de $P$. microphyllus se aislaron alcaloides con núcleo imidazólico como la Pilocarpina encontrado exclusivamente en plantas de este género (Sandhu et al., 2006) el cual es usado en procesos posoperatorios para promover salivación así como en el tratamiento del Glaucoma, enfermedad que causa la pérdida total de la visión (Cuca y Avila, 2007), así como la epiisopiloturina con actividad anticonceptiva y anti-inflamatoria (Silva et al., 2013). Del extracto etanólico de las hojas de $P$. alvaradoi se han aislado diversos metabolitos entre ellos lactonas como la Benzoil- $y$-valerolactona; triterpenos pentacíclicos conocidos como lupeol, y epibetulina; furanocumarinas como bergapteno, psoraleno y xantotoxina (Cuca y Avila, 2007).

Estudios recientes muestran que las sustancias naturales se han convertido en una alternativa terapéutica para el tratamiento de algunas enfermedades comunes como la leishmaniasis y el estrés oxidativo. La leishmaniasis constituye un grupo de enfermedades parasitarias, endémicas en 98 países, que afecta anualmente a 1,3 millones de personas y es la segunda infección parasitaria en el mundo con alrededor de 50.000 muertes anuales (Alvar et al., 2012). En Colombia, durante el año 2016 se han registrado 8679 casos de leishmaniasis (Instituto Nacional de Salud, 2016) y hasta el mes de mayo del año 2017 han ingresado al Sivigila 2364 casos de leishmaniasis, 18 de leishmaniasis mucosa, 31 de leishmaniasis visceral (15 confirmados y 16 probables) y 2315 confirmados de leishmaniasis cutánea, siendo la leishmaniasis cutánea la forma clínica más prevalente en el país (Instituto Nacional de Salud, 2017) y se estima que el 91\% de los municipios en Colombia que se encuentran por debajo de 1750 m.s.n.m han presentado casos de leishmaniasis (Segura et al., 2013).

A pesar de la utilización de tratamientos a base de antimoniales pentavalentes (Estibogluconato de sodio y el antimoniato de N-metilglucamina), Mitelfosina y Anfotericina B (Pulido et al., 2012), los casos de leishmaniasis se han ido incrementando considerablemente, demostrando ineficacia de estos medicamentos, razón por la cual se han modificado los tratamientos con aumentos de la dosis, conllevando a trastornos clínicos como efectos adversos. (Marin et al., 2016). Por otro lado, las enfermedades producidas por la acción de la alta producción de radicales libres (agentes oxidantes) asociados a malos hábitos alimenticios, bajos recursos económicos o la mala alimentación son causantes de diversas patologías como envejecimiento, arterosclerosis, cáncer, la enfermedad de Alzheimer, diabetes mellitus y el síndrome de estrés respiratorio (Cardona, 2013). Basados en los estudios realizados queda demostrado que el género Pilocarpus presenta un importante grupo actividades biológica, por lo tanto, la especie $P$. alvaradoi se constituye en un excelente ejemplar para la búsqueda de nuevos principios activos que presenten actividad antioxidante y leishmanicida mediante el desarrollo del estudio químico y la evaluación de la actividad antioxidante y leishmanicida de esta especie.

\section{METODOLOGÍA}

La metodología se presenta en dos secciones: en una se describe el procedimiento químico de obtención de metabolitos secundarios de la especie vegetal y en la otra sección se describe la evaluación de la actividad antioxidante y leishmanicida de los extractos

\section{Sección 1. Obtención de metabolitos secundarios}

Se describe el proceso desde la recolección del material vegetal, detallando los procesos de extracción, fraccionamiento e identificación de las sustancias extraídas. 


\section{Recolección del material vegetal}

La muestra de la especie Pilocarpus alvaradoi, fue colectada en el 2012 en el corregimiento de Santa Isabel del municipio de Montería (Córdoba). Un ejemplar de la muestra reposa en el Herbario de la Universidad de Córdoba con los números de colección HUC-004975 y 004976.

\section{Obtención de extractos}

Los extractos etanólicos y subextractos se obtuvieron por partición líquido-líquido del extracto crudo y posteriormente su fraccionamiento por cromatografía en columna; en ambos casos se utilizaron como solventes y reactivos: etanol, metanol, diclorometano, cloroformo, n-hexano, acetato de etilo, bencina de petróleo y sulfato de sodio anhidro. Los extractos primarios se obtuvieron por concentración a presión reducida utilizando un Rotaevaporador Büchi R-114. Los ensayos de actividad antioxidante se realizaron midiendo la absorbancia en un espectrofotómetro Génesis 20 thermo spectronic modelo 4001/4. El fraccionamiento por cromatografía en columna (CC) se hizo en tubos de vidrio de diferentes dimensiones, empacados con sílicagel $60 \mathrm{~F}_{254}$ de 0.063-0.2 mm. La visualización de las manchas se realizó empleando vapores de yodo $\left(\mathrm{I}_{2}\right)$, lámpara ultravioleta (UV) Camag $\lambda: 254 \mathrm{~nm}$ y $366 \mathrm{~nm}$. Se monitorearon las fracciones por cromatografía en capa delgada (CCD) con cromatoplacas de sílica-gel $60 \mathrm{HF}_{254}$ (Merck®) y para la cromatografía en capa delgada preparativa (CCDP) cromatoplacas de sílica gel $60 \mathrm{HF}_{254}$ de $1 \mathrm{~mm}$ (Merck®). Para el análisis por CGEM se utilizó un cromatógrafo de gases acoplado a un espectrómetro de masas (GC-MS) (PerkinElmer). La caracterización se realizó en base a los patrones de fragmentación y por comparación de la librería de espectros de masas NIST 8.0 y Wiley 8.0. Los espectros $\mathrm{RMN}^{-1} \mathrm{H}$ y ${ }^{13} \mathrm{C}$ fueron realizados en un equipo Bruker space de $300 \mathrm{MHz}$ usando $\mathrm{CDCl}_{3}$ como disolvente. Los puntos de fusión se tomaron utilizando un fusiómetro digital IA-9100 Electrothermal.

\section{Extractos etanólicos}

Las hojas, corteza y madera del material vegetal seco y molido, se sometieron a extracción por percolación con etanol (EtOH) al $96 \%$ a temperatura ambiente. El solvente fue retirado a presión reducida obteniéndose así los correspondientes extractos etanólicos.

\section{Fraccionamiento de extractos etanólicos de hojas y corteza}

Los extractos etanólicos de hojas y corteza de Pilocarpus alvaradoi fueron fraccionados por reparto utilizando solventes de polaridad creciente, Benzina de petróleo (BDP), cloroformo $\left(\mathrm{CHCl}_{3}\right)$, acetato de etilo (AcOEt), butanol $(\mathrm{BuOH})$ y agua $\left(\mathrm{H}_{2} \mathrm{O}\right)$.

\section{Fraccionamiento del extracto clorofórmico de hojas}

3 gramos de este subextracto de $\mathrm{CHCl}_{3}$ fueron fraccionados por $\mathrm{CC}$ soportado en silica gel $(120 \mathrm{~g})$ iniciando el proceso de elución con BDP:Diclorometano $\left(\mathrm{CH}_{2} \mathrm{Cl}_{2}\right)-1: 9$ y aumentando la polaridad en forma creciente hasta $\mathrm{CH}_{2} \mathrm{Cl}_{2}: \mathrm{MeOH}-9: 1$, obteniéndose 277 fracciones $(50 \mathrm{ml} / \mathrm{u}$ ) y posteriormente reunidas en 31 fracciones según el perfil cromatográfico mostrado desde A-1 hasta A-31. En la fracción A-8 se observó un sólido cristalino, los cristales fueron lavados con BDP y un posterior análisis por CCD de los cristales permitió observar una sola mancha denominado compuesto 1. La fracción A-15 (224,5 mg) fue sometida a CC con un sistema de elución $\mathrm{CH}_{2} \mathrm{Cl}_{2}$ :Metanol - 98:2 y aumentando su polaridad con metanol. De este proceso cromatográfico se obtuvieron 27 fracciones; un monitoreo por CCD permitió agruparlas en 12 nuevas fracciones desde A-15-1 hasta A-15-12. Un análisis por CCD para la fracción A-15-6 (15,1 mg), mostró una sola mancha, lo que evidenció la presencia de un compuesto puro al cual se le realizó un estudio espectroscópico completo y se designó como compuesto 2.

\section{Fraccionamiento del extracto clorofórmico de corteza}

De este extracto $943,0 \mathrm{mg}$ se fraccionaron por $\mathrm{CC}$ soportados en sílica gel y eluyendo con $\mathrm{CH}_{2} \mathrm{Cl}_{2}: \mathrm{CH}_{3} \mathrm{OH}$ 9:1, colectándose 12 fracciones de B-1 a B-12. El análisis por CCD y el peso de la fracción permitió selecionar la fracción B-10 y someterla a CC repetitiva eluyendo con BDP:AcOEt-3:7, de las 10 fracciones obtenidas, la fracción B-10-7 se sometió a CCDP, usando como sistema de elución BDP:AcOEt-3:7, obteniéndose una sustancia según el perfil mostrado por CCD, denominado compuesto 3 con un peso de $15.3 \mathrm{mg}$.

\section{Fraccionamiento del extracto etanólico de madera}

3 gramos de este extracto fueron sometidos a CC soportados sobre sílica gel $(120 \mathrm{~g})$ eluyendo con BDP: $\mathrm{CH}_{2} \mathrm{Cl}_{2}-1: 9$ y aumentando su polaridad hasta $\mathrm{CH}_{2} \mathrm{Cl}_{2}: \mathrm{MeOH}-8: 2$, de la cual fueron reunidas 38 fracciones 
según el perfil cromatográfico mostrado desde C-1 hasta C-38, teniendo en cuenta el análisis en CCD, las características de las manchas y el peso. La fracción C-11 (32.6 mg) mostró tres manchas separadas con buena resolución la cual fue sometida a CCDP obteniéndose dos sustancias denominados compuesto 4 y 5 que presentaron pesos muy pequeños y fueron sometidos posteriormente a Espectrometría de Masas (EM) para su identificación. La fracción C-13 (26,1 mg) presentó tres manchas una de ellas con mayor concentración fue sometida a CCDP eluyendo con $\mathrm{CH}_{2} \mathrm{Cl}_{2}: \mathrm{CH}_{3} \mathrm{OH}-99: 1$, de la que se obtuvo un sólido blanco denominado compuesto 6.

\section{Identificación de sustancias}

Los compuestos purificados fueron identificados utilizando las técnicas espectroscópicas IR, RMN-1 $\mathrm{H}, \mathrm{RMN}$ ${ }^{13} \mathrm{C}$, experimentos bidimensionales (COSY, HMQC, HMBC) y Cromatografía de gases acoplada a espectrometría de masa - CG-EM.

\section{Sección 2. Evaluación de la actividad antioxidante y leishmanicida de los extractos}

Se describe de manera detallada los ensayos de actividad antioxidante y leishmanicida sobre los extractos obtenidos.

\section{Ensayo de actividad antioxidante}

Se evaluó la actividad antioxidante de los extractos etanólicos y subextractos de la especie Pilocarpus alvaradoi (Rutaceae) empleando el método del radical libre DPPH• y el radical catiónico ABTS•+, siguiendo el método de Pastrana et al, 2016. Para el análisis estadístico de los resultados de la actividad antioxidante se utilizó como herramienta el programa Minitab 16 versión 5.1 y para la determinación del $\mathrm{IC}_{50}$ por regresión lineal, se utilizó el programa Statgraphics Plus versión 5.0, con un nivel de significancia del 95\% $(p<0.05)$.

\section{Ensayo de citotoxicidad y evaluación de la actividad leishmanicida}

En esta sección se describen los pasos desarrollados para llevar a cabo los procesos de evaluación de la citotoxicidad y de la actividad leishmanicida.

\section{Preparación de las soluciones de los extractos}

Para la evaluación de la actividad citotóxica se realizaron 6 diluciones dobles seriadas preparadas a partir de una solución madre de $200 \mu \mathrm{g} / \mathrm{ml}$ en dimetilsulfóxido (DMSO) (Sigma-Aldrich) obteniéndose seis soluciones (diluciones dobles seriadas) de concentración equivalente a 200.0, 100.0, 50.0, 25.0, 12.5 y $6.25 \mu \mathrm{g} / \mathrm{ml}$. Para la evaluación de la actividad leishmanicida inicialmente se preparó una solución a $20 \mu \mathrm{g} / \mathrm{ml}$. Y luego, para los extractos con alguna actividad a esta concentración se prepararon diluciones cuádruples seriadas a partir de $100.0,25.0,6.25$ y $1.562 \mu \mathrm{g} / \mathrm{ml}$. Para la anfotericina B se prepararon cuatro soluciones a partir de $1.0 \mu \mathrm{g} / \mathrm{ml}$. Todas las soluciones se prepararon en DMSO al 0.5\% y medio RPMI 1640 (Gibco) completo, es decir, suplementado con $10 \%$ de suero fetal bovino (SFB) (Gibco) y $1 \%$ de antibióticos (penicilina-estreptomicina) (Sigma).

\section{El parásito}

En los ensayos de actividad leishmanicida in vitro se utilizó la cepa de de L. (V) panamensis (UA140-pIR(-)eGFP) cultivados como promastigotes en medio bifásico NNN modificado (Novy-MacNeil-Nicolle), empleando como fase sólida agar sangre (Merck), fase líquida solución salina de buffer fosfato (PBS) más glucosa, pH 6.9 e incubados a $26^{\circ}$ C. Los promastigotes se usaron posteriormente para infectar los macrófagos U937 y obtener amastigotes intracelulares (ver abajo). Previamente a la infección in vitro de las células, se hizo un aspirado de la lesión del hámster con solución de fosfatos (PBS); posteriormente cultivada en medio de cultivo NNN e incubados a $27^{\circ} \mathrm{C}$ hasta obtener promastigotes.

\section{Actividad citotóxica sobre células U937}

La citotoxicidad se evaluó sobre las células U-937 empleando el micrométodo enzimático con bromuro de 3(4,5-dimetiltiazol-2-il)-2,5-difeniltetrazolio (MTT). Las células en fase exponencial de crecimiento se ajustaron a una concentración de 100.000 células/ml de medio RPMI-1640 suplementado con SFB y antibióticos. En un plato de 96 pozos se depositaron $100 \mu \mathrm{l}$ de células/pozo. Luego se adiciono en cada pozo 100 ul de cada una de las concentraciones del extracto. Como control negativo (viabilidad o no citotoxicidad) se usaron células en medio RPMI completo y mantenidas bajo las mismas condiciones de incubación, pero en ausencia de cualquier extracto; como control de citotoxicidad se utilizaron células expuestas a anfotericina B. Las células 
en presencia de los diferentes extractos, así como los controles se incubaron a $37^{\circ} \mathrm{C}$ en una atmósfera al $5 \%$ de $\mathrm{CO}_{2}$ durante 72 horas. Transcurrido el período de incubación, el efecto de los extractos sobre las células se determinó midiendo la actividad de la enzima mitocondrial succinato deshidrogenasa. Para ello, se adicionó, $10 \mu \mathrm{l} / \mathrm{pozo}$ de una solución de MTT a $5 \mu \mathrm{g} / \mathrm{ml}$ y se incubaron los platos a $37^{\circ} \mathrm{C}$ durante 3 horas. Pasado este período de incubación se adicionaron $100 \mu \mathrm{l} /$ pozo de una solución de $50 \%$ de isopropanol y $10 \%$ de dodecil sulfato de sodio (SDS) para solubilizar los cristales de formazán (Insuasty et al., 2015). Los platos se incubaron durante otros 30 minutos y la producción de formazán se midió en un lector de microplacas a $570 \mathrm{~nm}$. La citotoxicidad se determinó de acuerdo al porcentaje de disminución de la viabilidad y por ende, de la disminución en la cantidad de células obtenidas para cada extracto y la anfotericina B según las densidades ópticas (D.O) obtenidas en cada condición experimental. La disminución de la viabilidad celular denominada inhibición del crecimiento celular, se calculó utilizando los valores de D.O para cada condición usando la siguiente ecuación: \% Viabilidad = [D.O células expuestas al compuesto o control $\div$ D. O células no expuestas] $\times$ 100). Los valores de D.O obtenidas para las células en ausencia de extractos corresponde al 100\% de viabilidad o células vivas. Lego, con los porcentajes de viabilidad se calculó el porcentaje de mortalidad, que corresponde a 100 - \% viabilidad. Por último, con los porcentajes de mortalidad se calculó la concentración letal $50\left(\mathrm{CL}_{50}\right)$ calculada por el método de análisis dosis respuesta, Probit (Finney, 1971) usando el programa estadístico SAS Data Analysis (SAS Institute Cary NC, USA). Los ensayos se realizaron dos veces con tres réplicas para cada concentración evaluada. La citotoxicidad de cada extracto se clasificó según los valores de $C_{50}$ usando una escala propia: citotoxicidad alta $C_{50}<50 \mu \mathrm{g} / \mathrm{mL}$; citotoxicidad moderada: $\mathrm{CL}_{50}>50$ a $<200$ $\mu \mathrm{g} / \mathrm{mL}$ y citotoxicidad baja: $\mathrm{CL}_{50}>200 \mu \mathrm{g} / \mathrm{mL}$.

\section{Actividad sobre L. (V) panamensis}

La actividad de los extractos se evaluó sobre amastigotes intracelulares obtenidos luego de la infección in vitro de macrófagos U937 diferenciados con miristato acetato de forbol. Para ello, las células U937 mantenidas en cultivo en suspensión se centrifugaron a 1.500 rpm por 10 minutos y luego de descartar el sobrenadante, el botón de células se resuspendió a una concentración de 1X105 células/ml de medio RPMI 1640 al $10 \%$ SFB con $0.1 \mu \mathrm{g} / \mathrm{ml}$ de PMA. En cada pozo de una placa para cultivo celular de 24 pozos se dispersó $1 \mathrm{ml}$ de la suspensión de células y se incubaron a una temperatura de $37^{\circ} \mathrm{C}$ con una atmosfera al $5 \% \mathrm{CO}_{2}$. Transcurridas 48 horas de incubación las células se infectaron con promastigotes en fase estacionaria de crecimiento en una proporción 15:1 parásito: célula. Los platos se incubaron a $34^{\circ} \mathrm{C}$ en una atmosfera al $5 \%$ de $\mathrm{CO}_{2}$ durante 2 horas. Posteriormente se realizaron dos lavados con PBS para eliminar los parásitos libres, se adicionó un ml de medio RPMI 1640 y las células se incubaron nuevamente durante 24 horas. Pasado este tiempo, las células infectadas se expusieron a cada una de las concentraciones de los extractos durante 72 horas. Como control de infección se usaron células infectadas y cultivadas en ausencia de los extractos y como control de efectividad se empleó la anfotericina B. Luego de 72 horas de incubación a $34^{\circ} \mathrm{C}$ en una atmosfera al $5 \%$ de $\mathrm{CO}_{2}$ las células se removieron cuidadosamente del fondo del plato utilizando el émbolo de una jeringa y se analizaron en un citómetro de flujo leyendo a $488 \mathrm{~nm}$ de excitación y $525 \mathrm{~nm}$ de emisión con un láser de Argón (Galeano et al., 2012). Cada concentración de los extractos y de los controles se evaluó por triplicado en dos experimentos diferentes. La actividad anti-Leishmania se determinó de acuerdo a la carga parasitaria para cada concentración de cada extracto y anfotericina $\mathrm{B}$ según la intensidad media de flurescencia (IFM) (Pulido et al., 2012). Los valores de IFM obtenidas para las células infectadas en ausencia de extractos corresponde al $100 \%$ de parásitos. Para los compuestos que mostraron alguna inhibición a 20 $\mu \mathrm{g} / \mathrm{ml}$, se determinó también la concentración efectiva 50 (EC 50 ) calculada por el método Probit (Finney, 1971). Al igual que la citotoxicidad, la actividad anti-leishmania se clasificó según los valores de CL50, finalmente, la actividad citotóxica se correlacionó con la actividad anti-Leishmania calculando el índice de selectividad (IS) con la ecuación: $I S=\mathrm{CL}_{50} \div \mathrm{CE}_{50}$.

\section{RESULTADOS Y DISCUSIÓN}

De la especie Pilocarpus alvaradoi se aislaron seis compuestos (Fig. 1): una cumarina denominada Escopoletina, un alcaloide llamado Dictamina, tres esteroles conocidos como Lupeol, $\beta$-Sitosterol, Campesterol y el ácido oleico; las estructuras fueron asignadas en base al análisis de los datos espectroscópicos (RMN en una y dos dimensiones y espectrometría de masas) y por comparación con la literatura y mediante la búsqueda de artículos científicos.

Lupeol (1): Sólido blanco; p.f. 214-215 ํㅜ; IR: (KBr) $\lambda_{\max } 3250(\mathrm{OH})$ y $1643(\mathrm{C}=\mathrm{O}) \mathrm{cm}^{-1}$; EM m/z $426\left(\mathrm{M}^{+}\right)$; $\mathrm{RMN}^{1}{ }^{1} \mathrm{H}\left(400 \mathrm{MHz}, \mathrm{CDCl}_{3}\right) ; \delta_{\mathrm{H}}: 4,68(\mathrm{~d}, 1 \mathrm{H}), \delta_{\mathrm{H}}: 4.56(\mathrm{~d}, 1 \mathrm{H}), \delta_{\mathrm{H}}: 3.18(\mathrm{dd}, \mathrm{J}=5.4$ y J=10.8 Hz, $1 \mathrm{H}), \delta_{\mathrm{H}}: 2.36$ $(\mathrm{dt}, \mathrm{J}=10.4, \mathrm{~J}=5.7, \mathrm{~J}=1.0 \mathrm{~Hz}, 2 \mathrm{H}), \delta_{H}: 1.68(\mathrm{~s}, 3 \mathrm{H}), \delta_{H}: 1.03(\mathrm{~s}, 3 \mathrm{H}), \delta_{H}: 0.96(\mathrm{~s}, 3 \mathrm{H}), \delta_{H}: 0.94(\mathrm{~s}, 3 \mathrm{H}), \delta_{H}: 0.83$

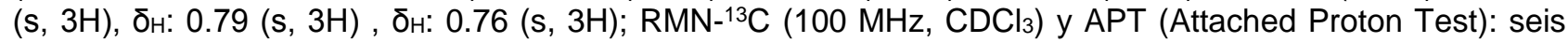
carbonos cuaternarios en $\delta_{c}: 151.03,43.04,42.86,40.86,38.90$ y 37.20 ppm; seis $\mathrm{CH}$ en $\delta_{H}: 79.04,55.32$, 50.46, 48.33, 48.02 y 38.08 ppm; once $\mathrm{CH}_{2}$ en $\delta_{H}: 40.03,38.73 ; 35.61,34.30,29.87,29.73,27.46 ; 25.16$, 20.96, 18.35 ppm y $\delta 109.36$ ppm característico de un carbono metilénico olefínico; siete $\mathrm{CH}_{3}$ en $\delta_{H}$ : 28.02 , 
19.34, 18.04, 16.15, 16.01, 15.40 y 14.58 ppm; COSY: $\delta 4.68$ con $\delta 4.56$ ppm, $\delta 3.18$ con $\delta 1.68$ ppm, $\delta 4.68$ con $\delta 1.68$ ppm (Laghari et al., 2011).

Escopoletina (2): Sólido amarillo; p.f. 206-207 ㄷ; IR: (KBr) $\lambda_{\max } 3460(\mathrm{O}-\mathrm{H}), 2920$ (C-H), 1700 (C=O), 1580, 1510, 1465 (C=C aromáticos) $\mathrm{cm}^{-1}$; EM m/z $192(\mathrm{M}+)$; RMN- ${ }^{-1} \mathrm{H}\left(300 \mathrm{MHz}, \mathrm{CDCl}_{3}\right) ; \delta_{\mathrm{H}}: 7.60$ (d, J= $\left.9.5 \mathrm{~Hz}, 1 \mathrm{H}\right)$ y $\delta_{H}: 6.27(\mathrm{~d}, \mathrm{~J}=9.5 \mathrm{~Hz}, 1 \mathrm{H})$ señales características de protones unidos a un anillo gama lactónico, $\delta_{H}: 6.92$ $(\mathrm{s}, 1 \mathrm{H}), \delta_{\mathrm{H}}: 6.85(\mathrm{~s}, 1 \mathrm{H})$ característicos de protones en un anillo aromático tetrasustituído; $\delta_{\mathrm{H}}: 3.97(\mathrm{~s}, 3 \mathrm{H},-$ $\left.\mathrm{OCH}_{3}\right) ; \mathrm{RMN}^{13} \mathrm{C}\left(75 \mathrm{MHz}, \mathrm{CDCl}_{3}\right.$ ) y DEPT-135: permitió evidenciar: cuatro carbonos cuaternarios oxigenados en $\delta_{c}: 161.4,150.2,149.7$ y 144.0 ppm; carbonos en la región aromática en $\delta_{c}: 143.2,113.3,111.5,107.7$, 103.2 ppm y una señal en la región alifática en $\delta_{c}: 56.3\left(-\mathrm{OCH}_{3}\right)$.

Dictamina (3): Sólido amarillo; p.f. 130-131 ${ }^{\circ} \mathrm{C}$; IR: (KBr) $\lambda \max 3108 \mathrm{~cm}^{-1}$ (C-H aromáticos), 2929, $2856 \mathrm{~cm}^{-1}$ (C-H, alifáticos), $1372 \mathrm{~cm}^{-1}$ (C-O); EM m/z 199 (M+); RMN-1 ${ }^{-1}\left(500 \mathrm{MHz}, \mathrm{CDCl}_{3}\right) ; \delta \mathrm{H}: 7.17$ (ddd, J=8.0, $1.2 \mathrm{~Hz}$, $1 \mathrm{H}$ ), $\delta \mathrm{H}: 7.45$ (ddd, J=8.0, $1.3 \mathrm{~Hz}, 1 \mathrm{H}$ ), $\delta \mathrm{H}: 7.77$ (dd, $\mathrm{J}=8.5 \mathrm{~Hz}, 1 \mathrm{H}$ ) y $\delta \mathrm{H}: 8.02$ (dd, $\mathrm{J}=8.4, \mathrm{~Hz}, 1 \mathrm{H}$ ) protones aromáticos, $\delta \mathrm{H}: 7.36(\mathrm{~d}, \mathrm{~J}=2.8 \mathrm{~Hz}, 1 \mathrm{H})$ y $\delta \mathrm{H}: 6.80(\mathrm{~d}, \mathrm{~J}=2.8 \mathrm{~Hz}, 1 \mathrm{H})$ protones de anillo furánico, $\delta \mathrm{H}: 4.17(\mathrm{~s}$, $3 \mathrm{H},-\mathrm{OCH} 3)$. RMN-13 $\mathrm{C}\left(125 \mathrm{MHz}, \mathrm{CDCl}_{3}\right)$ y DEPT-135: cinco carbonos cuaternarios en $\delta \mathrm{C}: 164.2,157.1$, 146.0, 119.0 y 103.7 ppm; seis $\mathrm{CH}$ en $\delta \mathrm{C}: 143.8,129.9,128.1,124.0,122.7$ y $105.1 \mathrm{ppm}$ y un $\mathrm{CH}_{3}$ en $\delta \mathrm{C}$ : $59.3(-\mathrm{OCH} 3)$.

Campesterol (4): Sólido blanco; p.f. 159-160 ํㅜ IR: $(\mathrm{KBr}) \lambda_{\max } 3600(-\mathrm{OH}), 3090(=\mathrm{C}-\mathrm{H}), 1670(\mathrm{C}=\mathrm{O}) \mathrm{cm}^{-1}$; EM: $\mathrm{m} / \mathrm{z} 400\left(\mathrm{M}^{+}\right), \mathrm{m} / \mathrm{z} 382\left(-\mathrm{H}_{2} \mathrm{O}\right), \mathrm{m} / \mathrm{z} 367\left(\mathrm{M}^{+}-\mathrm{CH}_{3}-\mathrm{H}_{2} \mathrm{O}\right) ; \mathrm{m} / \mathrm{z} 273\left(\mathrm{M}^{+}-\mathrm{CL}\right) ; \mathrm{m} / \mathrm{z} 255\left(\mathrm{M}^{+}-\mathrm{CL}-\mathrm{H}_{2} \mathrm{O}\right), \mathrm{m} / \mathrm{z} 231$, $\mathrm{m} / \mathrm{z} 213$. Los valores anteriores son consistentes con los datos comparados en la literatura (Choi et al., 2007).

Ácido oleico (5): Sólido blanco; p.f. $15 \stackrel{\circ}{\circ} \mathrm{C}$; IR: $(\mathrm{KBr})$ Umax $3510(\mathrm{O}-\mathrm{H}), 1726(\mathrm{C}=\mathrm{O}) \mathrm{cm}^{-1} ; \mathrm{EM}: \mathrm{m} / \mathrm{z} 282\left(\mathrm{M}^{+}\right)$, m/z $264\left(\mathrm{M}^{+}-\mathrm{H}_{2} \mathrm{O}\right), \mathrm{m} / \mathrm{z} 67\left[\mathrm{C}_{5} \mathrm{H}_{7}\right]^{+}, \mathrm{m} / \mathrm{z} 55\left[\mathrm{C}_{4} \mathrm{H}_{7}\right]^{+}, \mathrm{m} / \mathrm{z} 41\left[\mathrm{C}_{3} \mathrm{H}_{6}\right]^{+}$, los cuales corresponden a pérdidas características del compuesto. Los valores anteriores son consistentes con los datos comparados en la literatura (Lopez, 2009; Lopez, 2014).

Sitosterol (6): Sólido blanco; p.f. 135-136 ํㅜ; IR: (KBr) $\lambda_{\max } 3400(-\mathrm{OH}), 2980(\mathrm{C}-\mathrm{H}), 1700(\mathrm{C}=\mathrm{C}) \mathrm{cm}^{-1} ; \mathrm{EM} \mathrm{m} / \mathrm{z}$ $414\left(\mathrm{M}^{+}\right), \mathrm{m} / \mathrm{z} 396\left(-\mathrm{H}_{2} \mathrm{O}\right), \mathrm{m} / \mathrm{z} 381\left(\mathrm{M}^{+}-\mathrm{CH}_{3}-\mathrm{H}_{2} \mathrm{O}\right) ; \mathrm{m} / \mathrm{z} 273\left(\mathrm{M}^{+}-\mathrm{CL}\right) ; \mathrm{RMN}^{-1} \mathrm{H}\left(400 \mathrm{MHz}, \mathrm{CDCl}_{3}\right) ; \delta_{\mathrm{H}}: 3.52(\mathrm{~m}$, $1 \mathrm{H}, \mathrm{OH}), \delta_{\mathrm{H}}: 5.32(\mathrm{t}, 1 \mathrm{H}), \delta_{\mathrm{H}}: 1.01(\mathrm{~s}, 3 \mathrm{H}), \delta_{\mathrm{H}}: 0.68(\mathrm{t}, 3 \mathrm{H})$; RMN-13 $\mathrm{C}\left(100 \mathrm{MHz}, \mathrm{CDCl}_{3}\right)$ y DEPT-135: tres carbonos cuaternarios en $\delta_{H}: 140.8,42.3,36.5$ ppm; nueve $\mathrm{CH}$ en $\delta_{H}: 121.7,71.8,56.8,56.0,50.1,45.8$, 36.1, 31.9, 29.1 ppm; once $\mathrm{CH}_{2}$ en $\delta_{\mathrm{H}}$ : 42.3, 39.8, 38.3, 37.2, 33.9, 31.6, 29.7, 28.2, 24.3, 23.1, 21.1 ppm; y seis $\mathrm{CH}_{3}$ en $\delta_{\mathrm{H}}: 19.8,19.3,19.0,18.7,11.9,11.8 \mathrm{ppm}$. Los datos anteriores son consistentes con la formula $\mathrm{C}_{29} \mathrm{H}_{50} \mathrm{O}$ (Chaturvedula \& Prakash, 2012). Se pudo establecer que todas las sustancias aisladas de la especie Pilocarpus alvaradoi ya han sido aisladas de otros géneros como E. alata (Cuca et al. 2011), E. conspecta (Coy et al., 2013) y E. leicarpa (Liz et al., 2011) de la familia Rutaceae pero, se reportan por primera vez para esta especie (Fig 1).<smiles>C/C=C\C1CCC2(C)CCC3(C)C(CCC4C5(C)CC[C@H](O)C(C)(C)C5CCC43C)C12</smiles><smiles>CCC12CCC34CC[C@H](O)CC3=CCC4C1CCC2C(C)CCC(C)C(C)C</smiles>

4<smiles>COc1cc2ccc(=O)oc2cc1O</smiles>

2<smiles>CCCCCCCCCCCCCCC(=O)O</smiles>

5<smiles>COc1c2ccccc2nc2occc12</smiles>

3<smiles>CCC(CCC(C)C12CCCC3C4CC=C5CC(O)CCC5(C)C4CCC3C1CC2)C(C)C</smiles>

6

Fig. 1: Compuestos aislados de P. alvaradoi (Rutaceae).

Los extractos de la especie Pilocarpus alvaradoi mostraron ser activos frente a los radicales DPPH y ABTS ${ }^{\circ}$. Se evidenció que el extracto etanólico de corteza presentó una gran capacidad captadora de radicales libres 
frente a las especies radicalarias $\mathrm{DPPH}^{\cdot}$ y $\mathrm{ABTS}^{++}$, con un $\mathrm{IC}_{50}$ de $8.85 \mathrm{mg} / \mathrm{L}$ y $8.68 \mathrm{mg} / \mathrm{L}$ respectivamente; igualmente los subextractos de acetato de etilo de hojas y de madera mostraron una buena actividad antioxidante frente al radical $\mathrm{ABTS} \mathrm{S}^{+}$, con valores de $\mathrm{IC}_{50}$ de $6.55 \mathrm{mg} / \mathrm{L}$ y $9.23 \mathrm{mg} / \mathrm{L}$ respectivamente (Tabla 1), resultados que comparados con los valores de actividad antioxidante reportadas por Avila et al (2007) sobre el extracto etanólico de hojas de Pilocarpus alvaradoi el cual presentó muy buenos resultados mediante la aplicación del método TEAC con un valor de $1.51 \mathrm{mmol}$ trolox/g de extracto comparado con el ácido ascórbico con un valor de $1.01 \mathrm{mmol}$ trolox/g el cual fue usado como patrón, lo que deja en evidencia el efecto antioxidante de los metabolitos contenidos en este extracto, de igual forma para la especie Esenbeckia litoralis (Rutaceae) se reportan resultandos bastante similares a los obtenidos en los extractos de $P$. alvaradoi, ya que los extractos en acetato de etilo de corteza y hojas de $E$. litoralis mostraron valores de con un $\mathrm{IC}_{50}$ de 5.65 $\mathrm{mg} / \mathrm{L}$ y $7.65 \mathrm{mg} / \mathrm{L}$ respectivamente, frente al radical catiónico $\mathrm{ABTS}^{++}$, mientras que para el radical libre DPPH ${ }^{*}$ los extractos no reportan actividad antioxidante alguna (Marin et al., 2016-b) lo cual es consistente con lo reportado por Lasprilla y Cuca (2007) en su investigación sobre Esenbeckia runyonii cuyo extracto etanólico de corteza no mostró actividad antioxidante in vitro midiendo de manera indirecta la reducción del radical libre DPPH. Estos resultados muestran que los metabolitos que constituyen los subextractos en acetato de etilo de hojas y corteza de las especies $P$. alvaradoi poseen una mayor afinidad para disminuir los efectos oxidantes causados por el radical iónico ABTS•+. Lo que indica que en los extractos polares como el extracto etanólico y de acetato de etilo, se encuentran presentes los metabolitos secundarios con mayor capacidad antioxidantes constituidos por núcleos fenólicos (Ojito et al., 2012) como flavonoides, cumarinas y alcaloides, que son sustancias características en este género (Avila et al., 2007).

Tabla 1: Actividad antioxidante de extractos de P. alvaradoi (Rutaceae).

\begin{tabular}{|c|c|c|c|}
\hline \multirow{2}{*}{ Muestra } & \multirow{2}{*}{ Extractos } & \multicolumn{2}{|c|}{$\mathrm{IC}_{50}(\mathrm{mg} / \mathrm{L})$} \\
\hline & & $\mathrm{DPPH}^{\bullet}$ & $\mathrm{ABTS}^{\bullet+}$ \\
\hline \multirow{4}{*}{ Hojas } & $\mathrm{CH}_{3} \mathrm{CH}_{2} \mathrm{OH}$ & 97.76 & - . - . - - \\
\hline & BDP & $\ldots \ldots$ & 55.48 \\
\hline & $\mathrm{CHCl}_{3}$ & $\ldots \ldots$ & 33.46 \\
\hline & AcOEt & $\ldots \ldots$ & 6.55 \\
\hline Corteza & $\mathrm{CH}_{3} \mathrm{CH}_{2} \mathrm{OH}$ & 8.85 & 8.68 \\
\hline \multirow{3}{*}{ Madera } & $\mathrm{CH}_{3} \mathrm{CH}_{2} \mathrm{OH}$ & 48.86 & 14.40 \\
\hline & $\mathrm{CHCl}_{3}$ & $\ldots \ldots$ & 38.81 \\
\hline & AcOEt & $\ldots \ldots$ & 9.23 \\
\hline
\end{tabular}

La actividad in vitro de los extractos de hojas y corteza de $P$. alvaradoi frente a amastigotes intracelulares de L. (V) panamensis, UA140-pIR(-)-eGFP y células U937 se resumen en la Tabla 2. Los compuestos aislados no se evaluaron por que se aislaron en cantidades muy pequeña, que fueron usadas para su identificación por técnicas espectroscópicas. La actividad Leishmanicida se refleja en Concentración Letal 50 ( $\mathrm{CL}_{50}$ ), e índice de selectividad (IS) y la citotoxicidad en valores de Concentración Efectiva 50 (CE ( $_{50}$ ). Según la escala aquí establecida, la mayoría de los extractos, tanto de corteza como en hojas y madera mostraron citotoxicidad moderada, con valores de $\mathrm{CL}_{50}>50$ y $<200 \mu \mathrm{g} / \mathrm{ml}$, con excepción de los extractos en bencina de petróleo y diclorometano de corteza y el extracto en bencina de petróleo de hojas, que arrojaron citotoxicidad alta, con valores de $\mathrm{CL}_{50}<50 \mu \mathrm{g} / \mathrm{ml}$ (Tabla 2).

Tabla 2: Actividad citotóxica y leishmanicida in vitro de extractos de P. alvaradoi (Rutaceae).

\begin{tabular}{|c|c|c|c|c|}
\hline \multirow{2}{*}{ Muestra } & \multirow{2}{*}{ Extractos } & $\begin{array}{c}\mathrm{CL}_{50}(\mu \mathrm{g} / \mathrm{ml}) \\
\mathrm{X} \pm \mathrm{SD}\end{array}$ & $\begin{array}{c}\mathrm{CE}_{50}(\mu \mathrm{g} / \mathrm{ml}) \\
\mathrm{X} \pm \mathrm{SD}\end{array}$ & \multirow{2}{*}{ IS } \\
\hline & & U-937 & $\begin{array}{c}\text { Amastigotes } \\
\text { Intracelulares }\end{array}$ & \\
\hline \multirow{4}{*}{ Hojas } & $\mathrm{EtOH}$ & $83.7 \pm 17.5$ & $>83.7$ & $<1.0$ \\
\hline & BDP & $44.9 \pm 9.0$ & $>44.9$ & $<1.0$ \\
\hline & $\mathrm{CH}_{2} \mathrm{Cl}_{2}$ & $71.3 \pm 24.4$ & $>71.3$ & $<1.0$ \\
\hline & AcOEt & $50.4 \pm 5.4$ & $45.1 \pm 9.0$ & 1.1 \\
\hline \multirow{3}{*}{ Corteza } & $\mathrm{EtOH}$ & $70.5 \pm 14.2$ & $30.7 \pm 6.2$ & 2.3 \\
\hline & BDP & $32.1 \pm 5.0$ & $>32.1$ & $<1.0$ \\
\hline & $\mathrm{CH}_{2} \mathrm{Cl}_{2}$ & $38.0 \pm 0.9$ & $>38.0$ & $<1.0$ \\
\hline Madera & $\mathrm{EtOH}$ & $92.2 \pm 13.8$ & $>92.2$ & $<1.0$ \\
\hline Control & Anfotericina B & $54.1 \pm 11.7$ & $0.04 \pm 0.01$ & 1353 \\
\hline
\end{tabular}

Por su parte, la mayoría de los extractos no mostraron actividad contra amastigotes intracelulares de $L$. (V) panamensis, evidenciada por la incapacidad de inhibir la carga parasitaria ( $0 \%$ de inhibición a $20 \mu \mathrm{g} / \mathrm{ml}$ ). 
Solo el extracto en acetato de etilo de hojas y el extracto etanólico de corteza mostraron un buen potencial leishmancida reflejado en sus valores de Concentración Efectiva media $\left(C_{50}\right)$ de 45.1 y $30.7 \mu \mathrm{g} / \mathrm{ml}$ y un Índice de Selectividad de 1.1 y 2.3 respectivamente, frente al medicamento control Anfotericina $B$, cuya $C L_{50}$ es de $54.1 \mu \mathrm{g} / \mathrm{ml}$, comparando estos resultados con los reportados por Mafezoli et al (2000) se puede evidenciar la importancia de los compuestos presentes en las especies del género Pilocarpus contra parásitos protozoarios como la especie $P$. spicatus cuyo extracto etanólico del tallo presentó una gran capacidad para inhibir la carga parasitaria en un $97.3 \%$ sobre epimastigotes de Trypanosoma cruzi.

De igual forma investigaciones del extracto acetato de etilo de corteza de E. litoralis (Rutaceae) reportan una actividad pobre frente al parásito de $L$. (V) panamensis con un valor de $C_{50}$ de $78.26 \mu \mathrm{g} / \mathrm{ml}$, el cual según la escala establecida lo clasifica en el grupo de actividad baja, inhibiendo solo el 9.2\% de la carga parasitaria, mientras que el compuesto 1-hidroxi-3-metoxi- $N$-metilacridona aislado del extracto cloroformico de la corteza de E. litoralis si mostró actividad contra L. (V) panamensis, con una $\mathrm{CE}_{50}$ de $24.8 \mu \mathrm{g} / \mathrm{ml}$, lo que representa una capacidad para inhibir la carga parasitaria en un $37.2 \%$, de tal forma que presentar una $\mathrm{CE}_{50}<25 \mu \mathrm{g} / \mathrm{ml}$, permitió clasificarlo como un compuesto con alta actividad contra la especie Leishmania panamensis (Marin et al., 2016-a).

De esta manera se evidencia que el extracto etanólico de corteza de $P$. alvaradoi es el menos citotóxico con una $\mathrm{CL}_{50}$ de $70.5 \mu \mathrm{g} / \mathrm{ml}$, pero a su vez el más activo con una Concentración Efectiva media (CE ( $\left._{50}\right)$ de 30.7 $\mu \mathrm{g} / \mathrm{ml}$, y un IS de 2.3, lo que indica que este extracto tiene una mayor selectividad para inhibir la acción del parásito de L. (V) panamensis, comparado con el medicamento control Anfotericina B cuya CL50 es de 54.1 $\mu \mathrm{g} / \mathrm{ml}$. La actividad presentada por este extracto es atribuible a la presencia de compuestos fenólicos tipo alcaloides, lignanos, flavonoides y cumarinas, que han sido aislados en especies de este género (Pinheiro, 2002; Avila et al., 2007). Es importante destacar que el índice de selectividad (IS)>1 presentado por los extractos en acetato de etilo de hojas y etanólico de corteza de la especie $P$. alvaradoi lo convierten en una fuente interesante de metabolitos activos para realizar un posterior aislamiento y evaluación de las moléculas responsables de esta actividad.

\section{CONCLUSIONES}

Del estudio fitoquímico de las diferentes partes de $P$. alvaradoi (Rutaceae), se aislaron seis compuestos con diversidad molecular: una cumarina denominada Escopoletina, un alcaloide llamado Dictamina, tres esteroles conocidos como Lupeol, $\beta$-Sitosterol, Campesterol y el Ácido oleico.

El extracto etanólico de corteza presentó una gran capacidad captadora de radicales libres frente a las especies radicalarias DPPH• y $\mathrm{ABTS}^{\bullet+}$, con un $\mathrm{IC}_{50}$ de $8.85 \mathrm{mg} / \mathrm{L}$ y $8.68 \mathrm{mg} / \mathrm{L}$ respectivamente; igualmente los subextractos de acetato de etilo de hojas y de madera mostraron una buena actividad antioxidante frente al radical $\mathrm{ABTS}{ }^{\bullet+}$, lo que indica que en los extractos etanólico y de acetato de etilo están presentes metabolitos secundarios con capacidad antioxidante como: flavonoides, cumarinas y alcaloides que son sustancias características en especies de este género

El extracto en acetato de etilo de hojas y el extracto etanólico de corteza mostraron un buen potencial leishmancida reflejado en valores de Concentración Efectiva media (CE 50 ) de 45.1 y $30.7 \mu \mathrm{g} / \mathrm{ml}$ y un Índice de Selectividad de 1.1 y 2.3 respectivamente, comparado con el medicamento control Anfotericina B.

La actividad in vitro mostrada por estos extractos frente al parásito de $L$. (V) panamensis, aportan bases para continuar estudios tendientes a disponer de moléculas candidatas que pueda convertirse en una alternativa para el tratamiento de la leishmaniasis en Colombia y en el mundo.

\section{REFERENCIAS}

Alvar, J. y otros siete autores, Leishmaniasis worldwide and global estimates of its incidence, doi: 10.1371/journal.pone.0035671, PubMed Central, (en linea), 7(5), e35671 (2012)

Avila, M. y L.E. Cuca, Metabolitos fenólicos aislados de Pilocarpus alvaradoi (Rutaceae), Scientia et Technica: 13(33), 6769 (2007)

Beniddir, M.; L.E. Borgne y otros seis autores, Acridone Alkaloids from Glycosmis chlorosperma as DYRK1A Inhibitors, doi: 10.1021/np400856h, J. Nat. Prod. (en linea), 77(5), 1117-1122 (2014)

Cardona, W., S. M. Robledo., B. A. Rojano., F. A. Guarin., D. L. Muñoz y J. S. Saez, Actividad leishmanicida y antioxidante de extractos de Piper Daniel-gonzalezii Trel. (Piperaceae), Rev. Cubana de Plant. Med, 18(2), 268-277 (2013)

Chaturvedula, V. y I. Prakash, Isolation of Stigmasterol and $\beta$-Sitosterol from the dichloromethane extract of Rubus suavissimus, International Current Pharmaceutical Journal: 1(9), 239-242 (2012) 
Choi, J., E.O. Lee y otros ocho autores, Identification of Campesterol from Chrysanthemum coronarium L. and its Antiangiogenic Activities, doi: 10.1002/ptr.2189, Phytother. Res. (en linea), 21(10), 954-959 (2007)

Coy, C.A., L.E. Cuca y C. Quintero, Farmacognosia y Farmacobotánica de especies pertenecientes a los géneros Esenbeckia y Raputia (Rutaceae), Rev. Cubana de Plant. Med, ISSN 1028-4796 (en linea), 18(4), 638-653, 2013. http://scielo.sld.cu/pdf/pla/v18n4/pla15413.pdf. Acceso: 5 de Agosto (2004)

Cuca, L., C. Coy., E. Coy y J. Lozano, Actividad antibacteriana de terpenoides y alcaloides aislados de tres plantas colombianas, Rev. Cubana de Plant. Med, 18(4), 638-653 (2011)

Cuca, L y M. Avila, Nueva benzoil lactona y otros constituyentes de Pilocarpus alvaradoii (Rutaceae), Revista Colombiana de Química: 36(3), 285-290 (2007)

De Melo, M. y otros siete autores, Structures and Bioactivities of Dihydrochalcones from Metrodorea stipularis, doi: 10.1021/np500453x, J. Nat. Prod. (en linea), 77(11), 2418-2422 (2014)

Finney, J.D, Statistical logic in the monitoring of reactions to therapeutic drugs, Methods of Information in Medicine: 10(4), 237-245 (1971)

Galeano, E. y A. Martinez, Antiparasitic bromotyrosine derivates from carribbean marine sponge Aiolochoria crassa, Química Nova: 35(6), 1189- 1193 (2012).

Instituto Nacional de Salud, Boletín Epidemiológico No 44 de la Leishmania en Colombia, 2016, http://www.ins.gov.co/boletinepidemiologico/Boletn\%20Epidemiolgico/2016\%20Bolet\%C3\%ADn\%20epidemiol\%C3\%B3 gico \%20semana\%2044.pdf. Acceso: 13 de Noviembre (2016)

Instituto Nacional de Salud, Boletín Epidemiológico No 19, 2017, http://www.ins.gov.co/boletinepidemiologico/Boletn\%20Epidemiolgico/2017\%20Bolet\%C3\%ADn\%20epidemiol\%C3\%B3gico\%20semana\%2019.pdf.

Acceso: 26 de mayo (2017)

Insuasty, B y otros doce autores, An efficient synthesis of new caffeine-based chalcones, pyrazolines and pyrazolo[3,4b][1,4]diazepines as potential antimalarial, antitrypanosomal and antileishmanial agents, doi.org/10.1016/j.ejmech.2015.02.040, Eur. J. Med. (en línea), Chem, 93(1), 401-413 (2015)

Laghari, A., S. Memon., A. Nelofar y K. Khan, 2011. Alhagi maurorum: A convenient source of Lupeol, doi.org/10.1016/j.indcrop.2011.03.031, Industrial Crops and Products, (en línea), 34(1), 1141-1145 (2011)

Lasprilla, J y L. Cuca. Estudio Fitoquímico del Extracto Etanólico de la corteza de Esenbeckia runyonii (Rutaceae), Scientia et Technica: 13(33), 373-376 (2007)

Lin, T y otros seis autores, Prenylated Coumarins: Natural Phosphodiesterase-4 Inhibitors from Toddalia asiatica, doi: 10.1021/np401040d, J. Nat. Prod. (en línea), 77(4), 955-962 (2014)

Liz, R y otros siete autores, Protected effect of Esenbeckia leiocarpa upon the inflammatory response induced by carrageenan in a murine air pouch model, doi: 10.1016/j.intimp.2011.08.009, International Immunopharmacology, (en línea) 11(12), 1991-1999 (2011)

Lopez, J., J. Arrubla., G. Guerrero, Estandarización de Análisis de metilesteres de Ácidos Grasos por la Técnica de Cromatografía de gases acoplada a Espectrometría de masas, Scientia Et Technica, ISSN: 0122-1701, (en línea), 15(43), 228-233, 2009. http://www.redalyc.org/pdf/849/84917310040.pdf. Acceso: 5 de Agosto (2017)

López, A, Extracción, identificación y cuantificación de ácidos grasos presentes en las larvas y pupas del insecto comestible denominado "ticoco", Tesis de Magister. Dpto. de Ingenierías, Universidad Tecnológica de la Mixteca Oaxaca, Mexico (2014)

Mafezoli, J.; P. Vieir.; J, Fernandes. J.; M. Da Silva y S. Albuquerque, In vitro activity of Rutaceae species against the trypomastigote form of Trypanosoma cruzi, Journal of Ethnopharmacology, 73(1-2),335-340 (2000)

Marin, F.; O. Torres.; G. Santafé y S. Robledo, Estudio Fitoquímico y Evaluación del Potencial Leishmanicida de la Especie Esenbeckia litoralis (Rutaceae), doi: 10.4067/S0718-07642016000100017, Inf. Tecnol. (en línea), 27(1), 159-168 (2016a)

Marin, F.; O. Torres.; G. Santafé.; A. Saez y C. Guzman, Estudio Fitoquímico y Evaluación de la actividad antioxidante de Esenbeckia litoralis Donn.Sm. (Loro grande), Rev. Cubana de Plant. Med, ISSN 1028-4796 (en linea), 21(4), 1-12, 2016b. http://scielo.sld.cu/scielo.php?script=sci_arttext\&pid=S1028-47962016000400004. Acceso: 3 de Enero (2018)

Ojito, K.; Y. Herrera.; N. Vega y Portal, O, Actividad antioxidante in vitro y toxicidad de extractos hidroalcohólicos de hojas de Citrus spp. (Rutaceae), Rev. Cubana de Plant. Med, ISSN 1028-4796 (en linea), 17(4), 368-379, 2012. http://scielo.sld.cu/scielo.php?script=sci_arttext\&pid=S1028-47962012000400008. Acceso: 20 de Diciembre (2017)

Oliveira, A y otros seis autores, Chemical and Biological Investigations of Pilocarpus spicatus essential oils, Boletín Latinoamericano y del Caribe de Plantas Medicinales y Aromáticas, ISSN: 0717-7917 (en linea), 9(3), 206-211, 2010. http://www.ciencia.mx/ aceites. htm. Acceso: 5 de Agosto (2004)

Pastrana, O.; G. Santafé y J. Quirós, Actividad antioxidante del erizo de mar Mellita quinquiesperforata (Leske) e identificación de sus compuestos lipídicos mayoritarios, doi: 10.17533/udea.acbi.v38n104a02, Actualidades Biológicas, (en linea), 38(104), 15-22 (2016) 
Peng, J., R. Hartley, G. Fest y S. Mooberry, Amyrisins A-C, O-Prenylated Flavonoids from Amyris madrensis, doi: 10.1021/np200796e, J. Nat. Prod. (en linea), 75(3), 494-496 (2012)

Pinheiro, C, Extrativismo, cultivo e privatização do jaborandi (Pilocarpus microphyllus stapf ex holm.; Rutaceae) no maranhão, Brasil, Acta bot. Bras, 16(2), 141-150 (2002)

Pulido, S y otros siete autores, Improvement of the green fluorescent protein reporter system in Leishmania spp. for the in vitro and in vivo screening of antileishmanial drugs, doi: 10.1016/j.actatropica.2011.11.015, Acta Tropica, (en linea), 122(1), 36-45 (2012)

Sandhu, S., I. Abreu., C. Colombo y P. Mazzafera, Pilocarpine Content and Molecular Diversity in Jaborandi, Scientia Agricola: 63(5), 478-482 (2006)

Segura, R., A. Carrera., A. Cubides., A. Gomez y L. Perea, Falla Terapéutica en Leishmaniasis Valle del Cauca, Revista Medicina y Salud FUSM: 1(1), 30-32 (2013)

Shen, D y otros nueve autores, Constituents of the Roots of Clausena lansium and Their Potential Anti-inflammatory Activity, doi: 10.1021/np500088u , J. Nat.Prod. (en linea), 77(5), 1215-1223 (2014)

Silva, V y otros doce autores, Anti-inflammatory and Antinociceptive Activity of Epiisopiloturine, an Imidazole Alkaloid Isolated from Pilocarpus microphyllus, doi: 10.1021/np400099m, J. Nat. Prod. (en linea), 76(6), 1071-1077 (2013)

Uvarani, C., M. Sankaran., N. Jaivel., K. Chandraprakash., A. Ata y P. Mohan, Bioactive Dimeric Carbazole Alkaloids from Murraya koenigii, doi: 10.1021/np300464t, J. Nat. Prod. (en linea), 76(6), 993-1000 (2013)

Xia, $\mathrm{H}$ y otros siete autores, $A, D$-seco-Limonoids from the Stems of Clausena emarginata, doi: 10.1021/np400797s, J. Nat. Prod. (en linea), 77(4), 784-791 (2014) 